\title{
Silk as a Multifunctional Biomaterial Substrate for Reduced Glial Scarring around Brain-Penetrating Electrodes
}

\author{
Lee W. Tien, Fan Wu, Min D. Tang-Schomer, Euisik Yoon, Fiorenzo G. Omenetto, \\ and David L. Kaplan*
}

The reliability of chronic, brain-penetrating electrodes must be improved for these -neural recording technologies to be viable in widespread clinical applications. One approach to improving electrode reliability is to reduce the foreign body response at the probe-tissue interface. In this work, silk fibroin is investigated as a candidate material for fabricating mechanically dynamic neural probes with enhanced biocompatibility compared to traditional electrode materials. Silk coatings are applied to flexible cortical electrodes to produce devices that transition from stiff to flexible upon hydration. Theoretical modeling and in vitro testing show that the silk coatings impart mechanical properties sufficient for the electrodes to penetrate brain tissue. Further, it is demonstrated that silk coatings may reduce some markers of gliosis in an in vitro model and that silk can encapsulate and release the gliosis-modifying enzyme chondroitinase $A B C$. This work establishes a basis for future in vivo studies of silk-based brain-penetrating electrodes, as well as the use of silk materials for other applications in the central nervous system where gliosis must be controlled.

\section{Introduction}

Brain-interfacing technologies have great therapeutic potential, with applications ranging from direct neural control of prosthetic limbs ${ }^{[1]}$ to augmentation of sensory inputs. ${ }^{[2]}$ While first generation stimulatory neuroprosthetics, such as cochlear implants, have proven effective clinically, ${ }^{[3]}$ technologies that rely on chronic recording electrodes are still constrained by their lack of consistent performance. In many cases, penetrating recording electrodes can fail within weeks or months; ${ }^{[4-6]}$ this lack of long-term reliability must be improved to make these technologies viable for widespread use.

This inconsistent reliability is often attributed to gliosis, the inflammatory response in the central nervous system (CNS). ${ }^{[7,8]}$

L. W. Tien, Dr. M. D. Tang-Schomer,

Prof. F. G. Omenetto, Prof. D. L. Kaplan

Department of Biomedical Engineering

Tufts University

4 Colby St. Medford, MA 02155, USA

E-mail: david.kaplan@tufts.edu

F. Wu, Prof. E. Yoon

Department of Electrical Engineering

University of Michigan

2400 EECS Bldg., 1301 Beal Ave. Ann Arbor, MI 48109, USA

DOI: 10.1002/adfm.201203716
Gliosis is exemplified by the formation of a glial scar around the implanted electrode in an attempt by the body to isolate the injury site from healthy tissue. ${ }^{[9]}$ A dense accumulation of glial cells, including microglia and reactive astrocytes, act as a physical barrier between the electrode and targeted neuronal populations and this encapsulation can increase the electrical impedance at the recording site. ${ }^{[10]}$ Reactive glia produce extracellular matrix molecules, such as chondroitin sulfate proteoglycans (CSPGs), that inhibit axonal growth, preventing regeneration of damaged neurons near the electrode. As the scarring progresses, the electrode becomes incapable of recording neural signals.

The large mismatch between the elastic modulus of brain tissue and conventional silicon-based electrode shanks has been implicated as a significant factor contributing to chronic gliosis. Finite element analysis techniques have been used to model the large stress concentrations and "micromotion" effect that occur at the brain-electrode interface in response to indwelling, inelastic materials. ${ }^{[11,12]}$ Mechanical stress has been shown to stimulate astrocyte reactivity and neuronal death in vitro, ${ }^{[13]}$ and in vivo studies have found increased scarring and reduced neuronal density around less compliant probes..$^{[14-16]}$

One strategy to provide strain relief at the electrode-tissue interface is to use flexible, thin-film probes fabricated from polymers such as polyimide ${ }^{[17,18]}$ or parylene. ${ }^{[19-24]}$ Such flexible probes introduce an additional challenge, however, as these devices may be incapable of penetrating the surface of the brain to achieve insertion without buckling. Consequently, the incorporation of dissolvable, degradable or mechanically switchable materials has been proposed as a means to provide the necessary stiffness for insertion of the device, followed by a return to a flexible state in the hydrated brain tissue. Readily dissolvable materials such as polyethylene glycol, ${ }^{[20-22]}$ sugar, ${ }^{[25]}$ gelatin, ${ }^{[26]}$ polyglycolic acid, ${ }^{[27]}$ and tyrosine-based polymers, ${ }^{[28]}$ as well as a non-degradable composite of polyvinyl acetate and cellulose, ${ }^{[19,29]}$ have all been explored as stiffening materials for flexible electrodes.

Reduction of glial scarring around implanted probes has also been investigated from a biochemical approach. Studies have shown anti-inflammatory agents such as dexamethasone ${ }^{[30-34]}$ and alpha-melanocyte stimulating hormone ${ }^{[35]}$ reduce gliosis 
and improve neural recordings when released from the surface of penetrating electrodes. Other bioactive agents, such as the cell cycle inhibitor flavopiridol ${ }^{[36]}$ and neural cell adhesion molecule $\mathrm{L} 1^{[37]}$ have also been shown to reduce scarring and improve neuron densities around implanted probes, respectively.

Though not yet widely explored in the context of brain-penetrating electrodes, controlled delivery of molecular therapeutics used to reduce the effects of glial scarring following spinal cord injury ${ }^{[38]}$ may also prove useful for reducing the negative effects of gliosis around an electrode implant. The bacterial enzyme chondroitinase $\mathrm{ABC}$ (chABC) is well known to promote recovery after spinal cord injuries by degrading inhibitory CSPGs and allowing axons to grow across the glial scar. ${ }^{[39]}$ Delivery of the enzyme from a brain-penetrating electrode may provide a similar beneficial effect by removing barriers to axon growth and regeneration near the recording site. The in situ release of bioactive chABC has proven challenging due to the fact that the enzyme is unstable, degrading rapidly at physiologic temperatures ${ }^{[40]}$ but recent work has highlighted the utility of the sugar trehalose as a thermostabilizer for the enzyme. ${ }^{[41,42]}$

Here, we propose silk fibroin as a multifunctional material for fabricating brain-penetrating electrodes that combine dynamic mechanical properties with the capacity for local release of sensitive therapeutics. Silk is both biocompatible and biodegradable, ${ }^{[43,44]}$ with tunable, hydration dependent mechanical properties. ${ }^{[45]}$ Through controlled crystallization of the protein, it can be programmed to dissolve immediately upon exposure to liquid, or to persist for years in vivo. ${ }^{[46]}$ Silk can be processed into diverse material formats including fibers, films, gels and sponges using only water as a solvent and under ambient conditions, ${ }^{[47]}$ which is conducive to the encapsulation of labile molecules. ${ }^{[48]}$

In contrast, stiffening materials prepared via evaporation of chemical solvents ${ }^{[16,28]}$ may preclude the incorporation and release of sensitive biologics such as enzymes and growth factors. Furthermore, most previously proposed electrode coating materials dissolve within minutes after hydration, ${ }^{[21,22,25-27]}$ making it impossible to achieve extended drug release over days or weeks. Silk's tunable structure allows for control over therapeutic delivery rates. ${ }^{[49,50]}$

Recent work has highlighted the utility of silk as a biomaterial for various applications in the nervous system. Silkbased nerve guides have been extensively studied as scaffolds for regeneration in the peripheral nervous system, ${ }^{[51-56]}$ and silk has also been shown to support and direct the growth of various cell types from the CNS. ${ }^{[57,58]}$ In the brain, specifically, silk has been utilized to deliver adenosine to reduce epileptic seizures in rats ${ }^{[49]}$ and as a vehicle for enhancing conformal contact between a planar electrode array and the brain's surface. ${ }^{[59]}$

In this study, we fabricated and characterized silk coatings with properties that enable the delivery of mechanically dynamic thin-film penetrating probes to the brain. We used an in vitro model of glial scarring around a microwire electrode mimic to assess the CNS inflammatory response to silk. Finally, we tested the capacity for silk to encapsulate and release the sensitive enzyme chABC to reduce the axon growth inhibiting CSPG components of the glial scar.

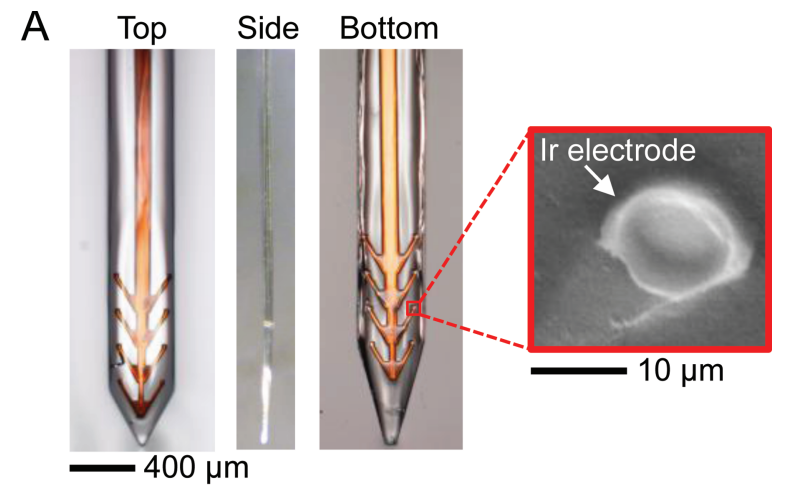

B

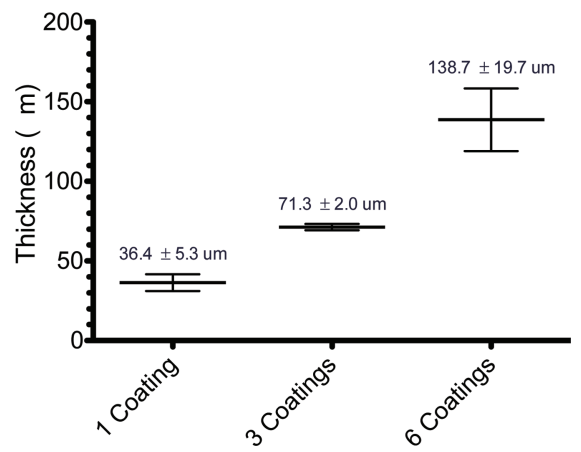

Figure 1. A) Light microscope images of flexible polyimide-based electrodes after coating in silk. Various tip geometries such as $60^{\circ}$ (Top view) and $30^{\circ}$ (Bottom view) angles can be fabricated based on the underlying mold. The single-side coating technique allows recording sites to remain exposed (SEM image, inset). B) Graph demonstrating control of device thickness based on the number of coatings of silk solution ( $N=3 /$ group). Error bars show standard deviation.

\section{Results}

\subsection{Application of Silk Coatings to Flexible Neural Probes}

A layer-by-layer casting technique was used to coat polyimide based, thin film neural probes with silk fibroin (Figure 1A). The probes were centered, with the electrode recording sites facing down, on polydimethylsiloxane (PDMS) molds that were fabricated using a laser cutter or soft lithography microfabrication techniques. The design of the underlying molds allowed for coatings with various dimensions and tip geometries (Figure 1A, Top, Bottom). Concentrated silk solution $(\approx 15 \%$ $\mathrm{w} / \mathrm{v}$ ) was applied to the molds by drawing a bead of solution from the tip of a pin along the length of the probe shank. This coating procedure produced silk coatings on only one side of the probe shank, allowing the recording sites on the probe to remain exposed (Figure 1A, inset).

The draw speed and viscosity of the silk solution, as well as the mold shape, dictated the amount of solution that adhered to the mold and probe. After drying, successive layers of silk were applied in the same manner in order to increase the thickness of the coating. For blunt tipped, $400 \mu \mathrm{m}$ wide molds, 3 layers of silk produced a final thickness of $\sim 70 \mu \mathrm{m}$ on average and 6 layers resulted in twice the thickness, $\sim 140 \mu \mathrm{m}$ on average (Figure 1B). 

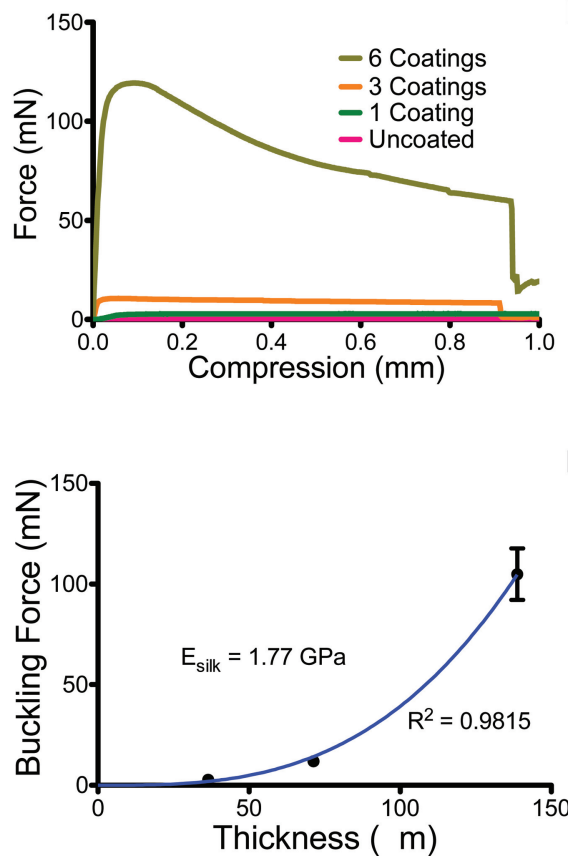

B
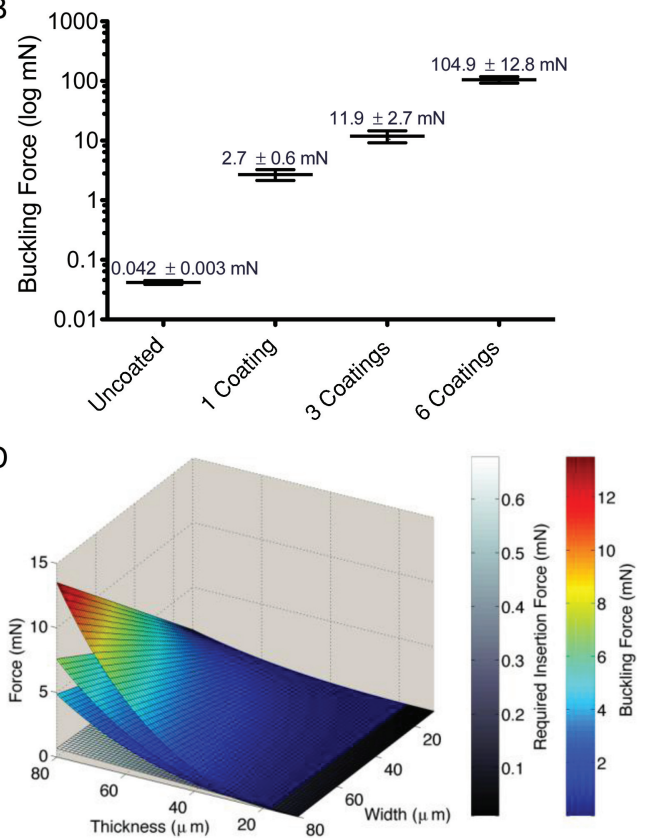

Figure 2. A) Representative force curves for uncoated, 1, 3, and 6 coated electrodes, fixed and driven perpendicularly onto a stage. B) Average buckling forces (log scale) for uncoated, 1, 3 and 6 coate-d electrodes ( $N=3$ /group). Error bars show standard deviation. C) Regression fit (blue line) of Euler's beam buckling equation to experimental force and thickness data allowing estimation of the elastic modulus of the silk coatings. D) Color surfaces: plots of the predicted buckling force for 3, 4, and $5 \mathrm{~mm}$ long silk shanks (longer shanks buckle at lower forces) using the elastic modulus derived in (C) and a flat tip with the given dimensions. Gray scale surface: Force required for insertion of a flat tipped probe with the given dimensions into mouse cortex (based on force data reported by Sharp et al. ${ }^{[60]}$ for a $100 \mu \mathrm{m}$ diameter wire inserted at $822 \mu \mathrm{m} / \mathrm{s}$ ).

\subsection{Mechanical Properties of Silk-Coated Neural Probes In Vitro}

Uncoated, 1 layer, 3 layer, and 6 layer silk-coated probes were mechanically characterized using a buckling test in which probes were driven perpendicularly onto a hard, flat surface at a rate of $0.5 \mathrm{~mm} / \mathrm{min}$. Representative force curves for each group are shown in Figure 2A. For all conditions tested, there was an initial linear increase in force while the probes remained straight before buckling. As the probes continued to be driven onto the surface, the shanks began to buckle and bend. This was characterized by the peak and gradual decrease in force observed for all samples. Eventually, the silk coating on the 3 and 6 layer probes fractured midway down the shank, resulting in a sharp decrease in the force curves.

For practical in vivo applications, neural probes must remain straight during insertion in order to reach a precisely targeted region of the brain. Therefore, the buckling force should not be exceeded during insertion into the cortex. The average buckling force was approximately $42 \mu \mathrm{N}$ for the uncoated probe, $3 \mathrm{mN}$ for the 1 layer silk-coated probe, $12 \mathrm{mN}$ for the 3 layer coated probe, and $105 \mathrm{mN}$ for the 6 layer coated probe (Figure 2B).

We modeled the buckling mechanics of the silk-coated electrodes using Euler's buckling equation for rectangular beams (Equation 1). Using non-linear regression with the elastic modulus of the electrode as the independent variable, Euler's equation was fit to a plot of the thickness and buckling force of the 1,3 , and 6 coated electrodes (Figure 2C). Assuming that the silk contributed the majority of the mechanical properties to the coated electrodes, the fit predicted the elastic modulus of silk $\left(E_{\text {silk }}\right)$ to be approximately $1.8 \mathrm{GPa}$ under ambient conditions.

This derived elastic modulus and Equation 1 were then used to generate $3 \mathrm{D}$ plots showing the predicted buckling force for blunt tipped silk shanks with various dimensions (Figure 2D, color plots). The three successive color surfaces show, from top to bottom, the predicted buckling force for 3, 4, and $5 \mathrm{~mm}$ long probes with thicknesses and widths specified on the $\mathrm{x}$ - and $\mathrm{y}$ axes. The theoretical force required to penetrate mouse brain tissue with an implant of a given cross-sectional area is also shown (Figure 2D, gray scale plot). This plot was calculated using Equation 2 and published force measurements taken during the insertion of a $100 \mu \mathrm{m}$ diameter blunt tipped wire into a mouse cortex after removal of the outer meninges. ${ }^{[60]}$

\subsection{In Vitro Brain Phantom Insertions of Silk-Coated Neural Probes}

Uncoated, 3 layer, and 6 layer silk-coated probes were inserted into an agar hydrogel mechanical brain phantom in order to test the efficacy of the silk coating for facilitating electrode insertion into the cortex. The electrodes were clamped to the Instron crosshead in the same manner as for the buckling force test and lowered onto the gel surface at a rate of $4 \mathrm{~mm} / \mathrm{min}$. The uncoated probe was unable to penetrate the surface of the gel (Figure 3A). The 3 layer silk-coated probe penetrated the gel surface, but bent and curled within the gel as the insertion 

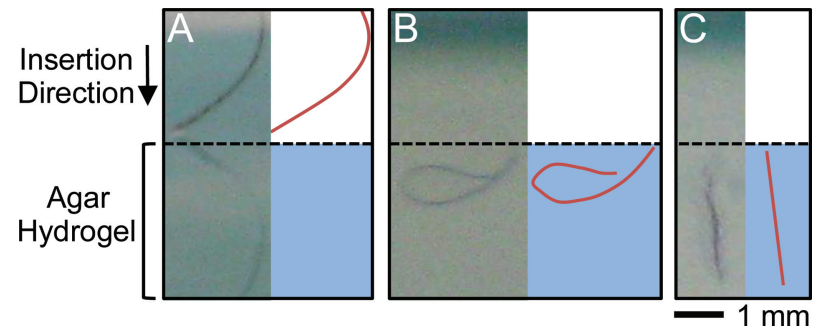

Figure 3. Images and accompanying cartoon representations showing insertions of A) uncoated, B) 3 coated, and C) 6 coated electrodes into a $0.6 \%$ agar hydrogel mechanical brain phantom. Dotted line depicts the surface of the hydrogel.

continued (Figure 3B). Finally, the 6 layer silk-coated probe penetrated the gel and remained straight during the entire insertion (Figure 3C).

\subsection{Response to Silk Coatings in an In Vitro Culture Model of Glial Scarring Around Microwire Electrodes}

The effect of silk fibroin coatings on reactive gliosis was tested using an in vitro model of glial scarring around an electrode mimic, which was previously used to assess the compatibility of various surface coatings. ${ }^{[61-63]}$ Uncoated and silk-coated (Figure 4A) segments of $50 \mu \mathrm{m}$ stainless steel microwire electrodes were dropped into day in vitro (DIV) 10 cultures of primary embryonic rat cortical cells. Serum and basic fibroblast growth factor (bFGF) were added to the culture to initiate the scarring response around the microwires ${ }^{[62]}$ and after one week
(DIV 17) the cultures were fixed and stained for the astrocyte marker glial fibrillary acid protein (GFAP) (Figure 4B, top), the microglia marker ionized calcium binding adapter molecule 1 (IBA1) (Figure 4B, middle) and chondroitin sulfate (CS), the primary component of CSPGs (Figure 4B, bottom).

Scarring around the uncoated and silk-coated wires was quantified (Figure 4C) based on the immunohistochemical staining (Figure 4B). Both the uncoated and silk-coated samples elicited a scarring response for all markers analyzed, as evidenced by areas of increased staining in close proximity to the wires relative to background levels far from the wires. A scar index was generated based on the intensity of staining between the edge of the microwire and a cutoff threshold determined relative to the background staining level in each image (see the Experimental Section). No significant difference was observed for the GFAP scar index around uncoated and silk-coated wires. However, the scar index was significantly less for both IBA1 $(p<$ $0.005)$ and CS $(p<0.05)$ around the silk-coated wires relative to the uncoated wire controls.

\subsection{Release of Bioactive chABC from Silk}

The encapsulation and release of the bacterial enzyme chABC was investigated as a potential approach to ameliorate axonal growth inhibition by CSPGs within the glial scar and to demonstrate the efficacy of silk materials for delivering sensitive therapeutics. ChABC (500 mU) and trehalose were bulk loaded into silk films, which were then water annealed to make them water insoluble. The films were placed in PBS at $37^{\circ} \mathrm{C}$ and enzyme activity in the buffer was tested over 7 days. ChABC released into the PBS consistently degraded approximately $80 \%$ of the
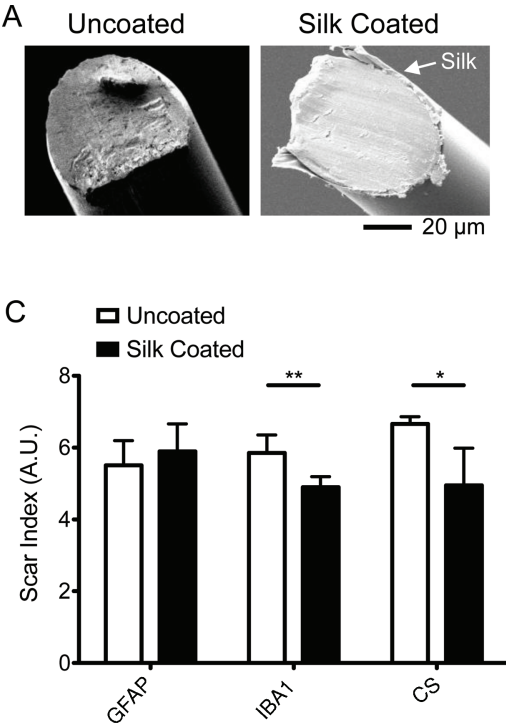

B
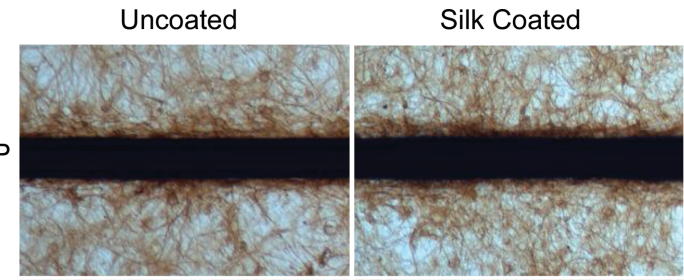

IBA1

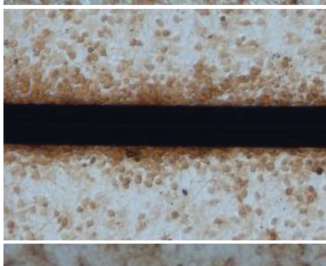

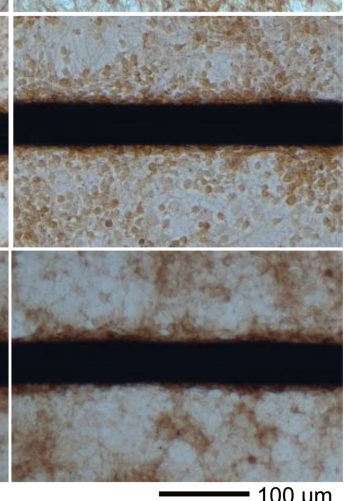

Figure 4. A) SEM images of uncoated (left) and silk-coated (right) stainless steel microwires. B) Representative images of staining for GFAP, IBAT, and CS around uncoated and silk-coated microwires dropped onto mixed neural cultures. C) Quantification of staining for GFAP, IBAI, and CS around the microwires. Number of images $(n)$ and wells $(N)$ analyzed per group were as follows: GFAP (uncoated: $n=116, N=14 ;$ silk-coated: $n=108, N=14$ ), IBAT (uncoated: $n=33, N=4$; silk-coated: $n=33, N=7$ ), CS (uncoated: $n=29, N=4$; silk-coated: $n=29, N=4$ ). Error bars show standard deviation. Horizontal bars denote a significant difference between staining around uncoated and silk-coated wires $(* p<0.05, * * p<0.005)$. 

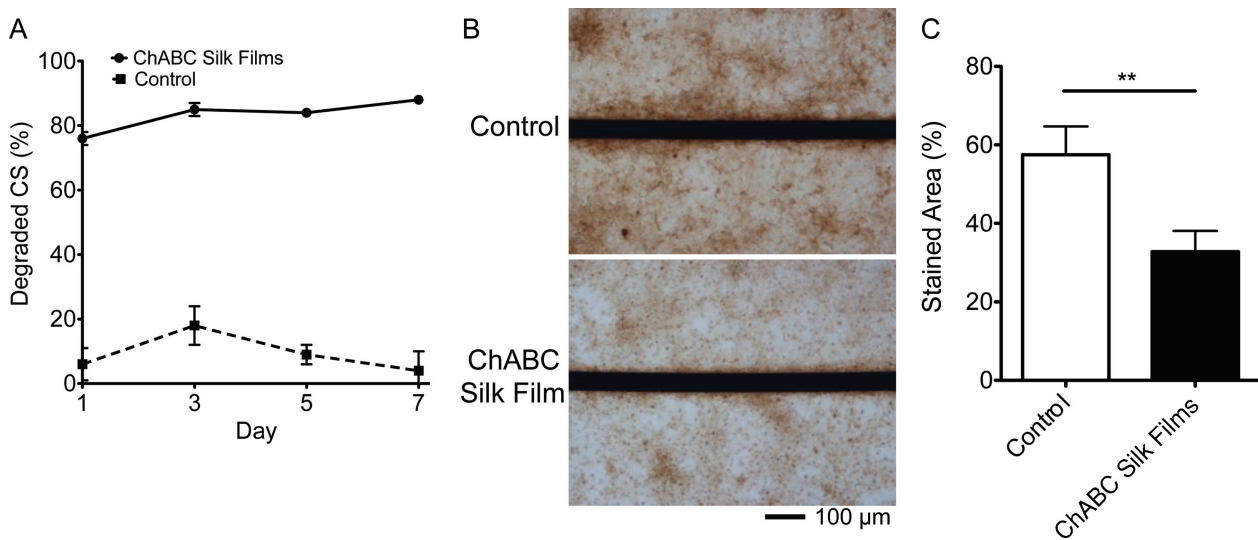

Figure 5. A) ChABC released from silk films maintained enzymatic activity for over 1 week. ( $N=4$ films/group) B) Representative images of staining for CS around microwires in cultures with blank (control) and chABC loaded silk films. C) Quantification of staining based on a defined intensity threshold. Number of images $(n)$ and wells $(N)$ analyzed per group were as follows: control $(n=21 ; N=3)$, chABC films $(n=17, N=3)$. Error bars show standard deviation. The horizontal bar denotes a significant difference between groups $(* * p<0.005)$.

CS substrate throughout the week (Figure 5A). This level of activity was similar to that observed when fresh $500 \mathrm{mU} / \mathrm{mL}$ chABC solution was tested. Silk films cast without chABC were used as a control; PBS from these wells had a negligible ability to degrade $\mathrm{CS}$.

The effect of chABC released from silk films was then tested in the in vitro model of glial scarring around a stainless steel microwire electrode. ChABC loaded silk films were floated on top of the culture medium in each well after the microwires were dropped onto the DIV 10 mixed neural cultures. Silk films without chABC were placed in the control wells. After one week (DIV 17), the films were removed and cultures were stained for CS (Figure 5B). The cultures containing the chABC silk films exhibited an almost 50\% reduction in CS staining (Figure $5 \mathrm{C}$ ).

\section{Discussion}

The biocompatibility of chronic brain-penetrating electrodes is integral to the long-term reliability and functionality of the device. The mechanical properties, innate immunogenicity, and chemical functionalization of the materials used to fabricate the probe shanks will all influence the tissue response in vivo. In this report, we demonstrated that silk possesses a unique combination of material properties that make it well suited for use in a penetrating electrode system.

Silk coatings were applied to pre-fabricated thin-film electrodes via layer-by-layer casting. The coating is conformal and the thickness is controllable based on the concentration of the silk solution and the number of layers applied. Because the casting steps are carried out using PDMS molds, the width and shape of the coating can be adjusted using various well-defined rapid prototyping and microfabrication techniques to pattern the underlying PDMS. The utility of the coating technique to a variety of geometries is important, as previous work has shown that the tip shape and surface and cross-sectional area of penetrating probes can affect the glial response. ${ }^{[8,64]}$ Additionally, in contrast to dipping-based coating methods, the casting technique produces a unilateral coating, allowing the recording sites on the underside of the probe to remain completely exposed. This should maximize the recording capabilities of the probe after implantation by ensuring that silk does not shield the recording sites from the targeted neurons.

Relative to the uncoated state, the layered silk coatings allow the flexible probes to sustain much greater forces before buckling when tested under ambient conditions. This difference in buckling forces is a result of both the increased modulus imparted by the silk coating, as well as the different dimensions of the coated probes that result from the underlying mold shape and layering of the silk. Because the buckling force is proportional to the cube of the device thickness, just a small change in thickness can produce a large change in the buckling force. This is evidenced by the fact that the 6 layer coated probes buckled at forces a full order of magnitude higher than the 3 layer coated probes, despite being only twice as thick (Figure 2B). The $E_{\text {silk }}$ of $1.8 \mathrm{GPa}$ predicted by the fit of Euler's beam buckling equation to the experimental data is on the same order of magnitude as previous reports of the modulus of dry silk films, ${ }^{[65]}$ providing validation for the use of this model to predict the buckling force for various silk-coated probe dimensions. As evidenced by the plots in Figure 2D, the predicted mechanical properties of silk shanks are more than sufficient to allow for penetration into brain tissue across a wide variety of dimensions.

It should be noted that the mechanical properties and buckling calculations for the devices in this study are on the conservative side of the full range of potential for silk materials. While, in this work, no post-coating treatment was applied to the silk devices, a variety of options are available to modulate the mechanical properties of the silk. Such treatments include additional pre-drying steps such as exposure to nitrogen gas ${ }^{[66]}$ or slow-drying, ${ }^{[67]}$ or exposure to methanol or water vapor to further alter the crystallinity. ${ }^{[45,68]}$

In addition, the comparison of the predicted buckling forces of the silk shanks with the force required to penetrate cortical tissue (Figure 2D) is also conservative because it assumes the use of blunt tipped devices. In practice, silk-based penetrating 
probes would be cast with angled tips (Figure 1A) in order to reduce the amount of force necessary to insert the device, as has been demonstrated in numerous studies. ${ }^{[60,69]}$ This technique allows for the insertion of smaller probes than would be predicted to be necessary based on calculations for blunt tipped devices. While features such as tapered tip geometries play a significant role in the buckling mechanics, the basic model provides a good point of reference for designing probe coatings with the necessary parameters to penetrate the brain.

The elastic modulus of hydrated silk films has previously been shown to be on the order of $20 \mathrm{MPa},{ }^{[45,68]}$ a decrease of nearly two orders of magnitude compared to the dry state measured in this work. This difference between dry and hydrated elastic modulus is similar to the mechanical properties demonstrated for other materials proposed for fabricating mechanically dynamic electrodes. ${ }^{[19]}$ The hydration-mediated transition in mechanics should reduce the strain concentration at the silk-coated electrode-tissue interface, improving long-term reliability. Indeed, the mechanically dynamic nature of the probes is evident from the agar insertion testing (Figure 3). While the 3 layer coated probe was stiff enough to penetrate the gel initially, during the course of insertion the tip of the probe hydrated before the shaft was fully inserted, causing bending within the gel. The thicker, 6 layer coated probe took longer to hydrate and transition to a more flexible state, allowing it to fully penetrate into the gel while remaining straight.

Based on these observations, relatively rapid insertion speeds will be important for future in vivo trials in order to allow the electrode shank to fully penetrate the brain before becoming too flexible due to hydration. This may be beneficial, as some studies have shown that faster insertion speeds may cause less acute damage to the brain. ${ }^{[70]}$ The hydration rate of the coatings may also be modulated by use of the previously discussed postcoating modification techniques to alter the molecular structure of the silk.

In addition to the dynamic mechanical properties, the cellular response to silk coatings in the in vitro model of glial scarring further supports its use in penetrating electrodes. Staining for GFAP, IBA1 and CS was increased around both silkcoated and uncoated microwires relative to background levels (Figure 4C). This is likely a result of both increased expression of the molecules of interest as well as an increased density of cells expressing those markers, as would be expected during a physiologic foreign body reaction in the brain. Silk coatings resulted in the same average amount of GFAP staining, a common marker for gliosis and astrocyte reactivity, relative to the uncoated inert stainless steel microwires, indicating that astrocyte proliferation and activation was similar in response to both materials. However, silk coatings stimulated significantly less IBA1 and CS staining around the microwire, suggesting that silk coatings may affect various aspects of gliosis independently. The decreased staining for IBA1 and CS around silk coatings indicates that silk may reduce the activation of microglia and production of inhibitory CSPG molecules during the glial scarring injury response relative to inert electrode implants.

While silk coatings alone appear to reduce some markers of gliosis when compared to stainless steel, the ability to encapsulate and release scar-inhibiting compounds provides an additional avenue for improving the chronic reliability of silk-based penetrating electrodes. The amenability of silk to controlled release strategies using a wide variety of therapeutics ${ }^{[50]}$ enables novel molecular interventions to the glial scarring process around penetrating devices to be tested. Research in this field has been relatively limited to date, with most approaches aimed at blunting the initiation of glial scarring around the implant with the use of anti-inflammatories such as dexamethasone. ${ }^{[30-34]}$ The focus on chABC in this study is based on developments in the field of spinal cord injury, where research has increasingly addressed not only the induction of glial scarring but also the inhibitory aspects of the scar ECM. ${ }^{[38]}$

We have demonstrated that chABC activity is maintained during loading and processing of silk materials and that release from silk devices is a viable way to deliver enzyme that remains active for at least 1 week at $37^{\circ} \mathrm{C}$ (Figure 5A). Further, chABC released from silk films was effective at reducing the amount of intact CS produced by cultured cortical cells in the glial scarring model over 1 week (Figure 5B,C). The all-aqueous processing conditions allowed the enzyme to be directly loaded into the silk device during a single fabrication step. This is advantageous compared to other chABC incorporation techniques, such as adsorption and immobilization, ${ }^{[71,72]}$ where loading amounts are unclear and subsequent release may be limited.

\section{Conclusions}

We have demonstrated that the innate mechanical properties of silk and low inflammatory potential, as well as its capacity to encapsulate and release sensitive gliosis-modifying compounds, make it an attractive candidate biomaterial for the next generation of chronic, indwelling neural probes. Such probes should have improved biocompatibility, resulting in reduced glial scar encapsulation and ultimately better long-term reliability. Future work will be directed at verifying this hypothesis in vivo through trial insertions and long-term implantation studies to assess and quantify gliosis around silk implants. This research has applications beyond penetrating electrodes, laying the groundwork for the use of silk fibroin for many CNS applications, such as in shunts and spinal cord nerve guides, where gliosis must be controlled at the tissue-implant interface to achieve optimal outcomes.

\section{Experimental Section}

Materials: All reagents were ordered from Sigma-Aldrich (St. Louis, MO), except where noted otherwise.

Preparation of Silk Solution: Silk solution was prepared from Bombyx mori silkworm cocoons according to the procedures described in our previous studies. ${ }^{[47]}$ Cocoons of B. mori silkworm silk were supplied by Tajima Shoji Co. (Yokohama, Japan). Briefly, the cocoons were degummed in a boiling $\mathrm{Na}_{2} \mathrm{CO}_{3}$ solution $(0.02 \mathrm{M})$ for $15 \mathrm{~min}$. The fibroin extract was then rinsed three times in DI water, dissolved in a $\mathrm{LiBr}$ solution $(9.3 \mathrm{M})$ yielding a $20 \% \mathrm{w} / \mathrm{v}$ solution, and subsequently dialyzed (MWCO $3,500 \mathrm{kDa}$ ) against DI water for 2 days to obtain regenerated aqueous silk fibroin solution (ca. $8 \% \mathrm{w} / \mathrm{v}$ ). The silk solution was concentrated to $\sim 15 \% \mathrm{w} / \mathrm{v}$ by leaving a dialysis cassette on the bench overnight.

Coating of Thin-Film Electrodes: Flat PDMS molds were prepared using two different techniques. For a rapid prototyping approach, 3-5 mm of Sylgard 184 (Dow Corning Corp., Midland, MI) was cast on petri 
dishes. After curing overnight at $60{ }^{\circ} \mathrm{C}$, the PDMS was cut into molds of varying design using a commercial laser cutter (Speedy 300 Laser Engraver, Trotec, Ypsilanti, MI). PDMS molds were also prepared using conventional soft lithography microfabrication techniques, ${ }^{[73]}$ which allowed for finer control of edge morphology and features. A dissecting microscope was used to manually align the electrodes (the fabrication of which has been detailed in a previous publication ${ }^{[74]}$ ) on the PDMS molds. The electrodes were coated by dipping the head of a pin into $15 \% \mathrm{w} / \mathrm{v}$ aqueous silk solution and drawing the bead of silk down the mold and electrode shank from the base to the tip, as a modification of our layer-by-layer deposition technique. ${ }^{[66]}$ Coatings were allowed to dry for 15 min before applying another layer.

Microscopy and Thickness Measurements: Coated probes were imaged using the 10x objective on a Zeiss Axiovert 40 CFL (Carl Zeiss AG, Germany) microscope. Thicknesses of probes coated using a $400 \mu \mathrm{m}$ wide blunt tipped mold were measured with QCapture Pro software. Scanning electron microscopy (SEM) was carried out using a Hitachi SU8000 Ultra-High Resolution SEM.

Buckling Force Test and Calculations: Coated $(400 \mu \mathrm{m}$ wide blunt tipped molds) and uncoated probes were tested under ambient conditions to determine their buckling force (the maximum force exerted by the probes when pinned and loaded in a vertical orientation). This characterization was chosen to mimic the type of loading experienced by the electrodes during insertion into the brain. A custom clamp was fabricated to fix the probes to the crosshead of an Instron 3366 mechanical testing frame (Instron, Norwood, MA). Samples were then lowered onto a fixed surface at a rate of $0.5 \mathrm{~mm} / \mathrm{min}$. Force data was captured using the $10 \mathrm{~N}$ Instron load cell, as well as with a Mettler-Toledo MS204S/03 analytical balance (Mettler-Toledo, LLC, Columbus, $\mathrm{OH}$ ) to confirm the accuracy of the load cell measurements. The balance was capable of reading mass to the $10^{\text {th }}$ of a milligram, allowing micro-Newton forces to be recorded with custom software that interfaced with the scale.

Euler's fixed-pin beam buckling equation: $[17,19]$

$F_{b}=\frac{\pi^{2} E_{s i l k} \frac{1}{12}\left(b h^{3}\right)}{(0.7 L)^{2}}$

was used to fit a curve to the measured buckling force and probe thicknesses using the least squares nonlinear regression algorithm in GraphPad Prism software (GraphPad Software, Inc., La Jolla, CA) with the elastic modulus as the independent variable. $F_{\mathrm{b}}$ is the buckling force, $E_{\text {silk }}$ is the elastic modulus (assuming negligible contribution from the polyimide film), $h$ is the thickness, $b$ is the width $(400 \mu \mathrm{m})$ and $L$ is the length $(5.5 \mathrm{~mm})$ of the probe.

Matlab software, version R2009b, (MathWorks, Natick, MA) was used to generate plots from Equation 1, with the elastic modulus derived from the nonlinear regression, that show the predicted buckling force for silk probes with varying dimensions. The force necessary to insert a probe of a given cross-sectional area into mouse cortical tissue was computed using Equation 2:

$F_{i}=p_{i} b h$

where $F_{i}$ is the insertion force, $p_{i}$ is the pressure required for insertion $h$ is the thickness of the probe, and $b$ is the width of the probe. $p_{i}$ was assumed to be $106 \mathrm{kPa}$ based on a reported maximum recorded force of $833.7 \mu \mathrm{N}$ for a $100 \mu \mathrm{m}$ diameter flat wire inserted into mouse cortex at a rate of $822 \mu \mathrm{m} / \mathrm{s}$ with the dura and pia removed. ${ }^{[60]}$

Brain Phantom Insertion Test: Insertion tests were performed using the Instron crosshead and custom clamp to lower coated and uncoated probes at a speed of $4 \mathrm{~mm} / \mathrm{min}$ onto $0.6 \%$ agar gel, a close match to the mechanical properties of the brain. ${ }^{[75]}$ The outcomes following insertion were photographed.

In Vitro Glial Scar Model Cell Culture: Glial scarring around silk-coated microwires was assessed using a 2D model based on the protocol developed by Polikov et al. ${ }^{[61,62]}$ Briefly, the cortices of embryonic day 18 rat pups were dissociated in trypsin $(0.5 \%)$ and plated in 24 well, poly-L-lysine coated plates at a density of 0.5 million cells/well. The cells were incubated at $37{ }^{\circ} \mathrm{C}$ in Neurobasal medium $(1 \mathrm{~mL})$ containing B27 supplement, pen-strep (1\%), GlutaMax (1\%, Invitrogen, Inc., Carlsbad, CA), and bFGF (10 ng, R\&D Systems, Minneapolis, MN). At DIV 10, the media was changed to include FBS (10\%, Invitrogen) and 2 segments of steel microwire, prepared as detailed below, were allowed to sink to the bottom of each well and rest on the dissociated brain cell cultures.

Cultures with wires were fixed at DIV 17 by incubating in paraformaldehyde (4\%) for $30 \mathrm{~min}$. After washing with PBS, cells were permeablized and blocked with PBS containing goat serum $(4 \%$, Invitrogen) and Triton-X (0.1\%). Primary antibodies for GFAP (mouse anti-GFAP), IBA1 (rabbit anti-IBA1, Wako Chemicals USA, Richmond, $V A$ ), and CS (mouse anti-CS) were diluted in permeablization buffer $(1: 1000)$ and incubated with the cells overnight at $4{ }^{\circ} \mathrm{C}$. After three PBS washes, cells were incubated with horseradish peroxidase conjugated secondary IgG antibodies specific for rabbit and mouse (Santa Cruz Biotechnology, Santa Cruz, CA), diluted in permeablization buffer (1:500), for at least $2 \mathrm{~h}$. Color was developed using a DAB substrate kit according to the manufacturer's instructions and incubated with cells for $1 \mathrm{~h}$.

Steel Microwire Coating: Stainless steel electrode microwire with a diameter of $50 \mu \mathrm{m}$ (A-M Systems, Sequim, WA) was used for the scarring assay. Silk coatings were achieved by dipping the microwire into silk solution $(\approx 7 \% \mathrm{w} / \mathrm{v})$, allowing the silk to dry on the wire in a $60{ }^{\circ} \mathrm{C}$ oven for $15 \mathrm{~min}$, and then water annealing for $20 \mathrm{~min}$ to crystallize the silk. The dipping procedure was repeated 5 times in order to build up an approximately $2 \mu \mathrm{m}$ thick coating of silk around the circumference of the wire. SEM images of uncoated and silk-coated wires were taken using an EVO10 microscope SEM (Carl Zeiss AG).

Glial Scar Model Analysis and Quantification: After fixing and staining cells, images were taken along the entire length of each microwire using a 10x objective on a Zeiss Axiovert 40 CFL microscope. The images were analyzed in an automated fashion using custom Image) (NIH, Bethesda, MD) and Matlab scripts based on the methods of Polikov et al. ${ }^{[62]}$ Briefly, pixel intensity was averaged across the entire image with the microwire registered on the horizontal. The intensity values were then normalized and the position of the microwire was determined to be the $50 \mu \mathrm{m}$ region with the lowest pixel intensity values. The background staining level was determined by averaging the pixel intensity of the $100 \mu \mathrm{m}$ region farthest from the wire at each edge of the image. Finally, the scar index for each wire was defined as the area enclosed between the edge of the microwire and the averaged pixel intensity curve at the point where the intensity reached $90 \%$ of the previously determined background value on each side of the wire. In this way, the scar index provides a quantitative means to assess the intensity of staining proximal to the microwires.

The scar index was calculated for each well by averaging the scar indexes of each image taken in the well. The scar indexes for all wells stained for a given molecule were averaged and used for statistical analysis and comparisons. For GFAP, 14 wells were analyzed with silkcoated wires and 14 wells were analyzed with uncoated wires. For IBA1, 7 wells were analyzed with silk-coated wires and 4 wells were analyzed with uncoated wires. For CS, 4 wells were analyzed with silk-coated wires and 4 wells were analyzed with uncoated wires.

Fabrication of ChABC Silk Films: Silk films containing chABC were prepared by modifying a commonly used casting technique developed in our lab. ${ }^{[76,77]}$ Briefly, chABC $(5 \mathrm{U})$ was dissolved in DI water $(250 \mu \mathrm{L})$ containing trehalose $(1 \mathrm{M})$. Next, the chABC solution $(100 \mu \mathrm{L})$ was added to silk solution ( $300 \mu \mathrm{L}, 6.7 \% \mathrm{w} / \mathrm{v})$ to yield silk solution $(400 \mu \mathrm{L}, 5 \% \mathrm{w} / \mathrm{v})$ containing chABC $(2 \mathrm{U})$ and trehalose $(250 \mathrm{mM})$. Films were cast by spreading the silk/chABC solution $(100 \mu \mathrm{L})$ onto $14-\mathrm{mm}$ diameter PDMS disks and air drying overnight. The next day, the films were made water insoluble by annealing in a water filled dessicator at $24 \mathrm{~mm} \mathrm{Hg}$ for $3 \mathrm{~h}$. Identical films were made without chABC to serve as controls.

Activity of chABC Released from Silk Films: ChABC-loaded and blank control films were placed into wells of a 24-well plate containing PBS $(1 \mathrm{~mL})$ in a $37^{\circ} \mathrm{C}$ incubator. On days $1,3,5$, and 7, PBS $(10 \mu \mathrm{L})$ was removed from each well and assayed for activity using the DMMB assay detailed below. The amount of degraded CS was averaged and plotted for 4 replicates each of the chABC-loaded and blank control films. 
Dimethylmethylene Blue (DMMB) Assay for ChABC Activity: The catalytic activity of chABC was assessed by measuring the amount of intact disaccharide after incubating a solution of chABC with CS. Briefly, a solution of CS in PBS $(10 \mu \mathrm{L}, 200 \mu \mathrm{g} / \mathrm{mL})$ was mixed with PBS $(10 \mu \mathrm{l})$ collected from the silk films floating in PBS. The solution was incubated at $37^{\circ} \mathrm{C}$ in a 96 well plate for $1 \mathrm{~h}$. Next, DMMB color reagent $(180 \mu \mathrm{L})$, consisting of DMMB dye (16 mg), glycine $(3.04 \mathrm{~g}), \mathrm{NaCl}(2.37 \mathrm{~g})$ and $\mathrm{HCl}(95 \mathrm{~mL}, 0.1 \mathrm{M})$ dissolved in $\mathrm{DI}$ water $(1 \mathrm{~L}),{ }^{[78]}$ was added to the sample. The $A_{530}$ was read immediately using a SpectraMax M2 plate reader (Molecular Devices, LLC, Sunnyvale, CA). Absorbance values were converted to CS concentrations based on a previously constructed standard curve.

Effect of ChABC Delivery in the Glial Scar Model: The glial scar model protocol was followed as detailed previously. At DIV 10, chABC-loaded and blank silk films were floated on top of the medium in each well after uncoated microwires were dropped onto the cells. The cultures were fixed and stained for CS at DIV 17. Images were taken along the microwires in each well using identical exposure settings. Image) was then used to set a universal intensity threshold and quantify the area of each image with staining above the threshold level. Stained area percentages were averaged for all images in each well and these values were then used for statistical comparisons. Three wells were analyzed for each condition.

Statistical Analysis: Data is presented in graphs as average \pm standard deviation. Average values were obtained from three separate replicates, except where otherwise noted. Data was analyzed for significance with GraphPad Prism software using a two-tailed unpaired t-test.

\section{Acknowledgements}

This work was supported by the NIH P41 Tissue Engineering Resource Center (EB002520) and the AFOSR. The authors thank Gregory Frost, Dr. Yasuko Nakamura and the Steven J. Moss Lab in the Tufts University Neuroscience Department for providing the dissected rat cortices. The authors also thank Prof. Gary Leisk, Maria Arshanskiy, Tim Lo, and Ben Partlow for assistance with the laser cutter, electrode coating and mechanical characterization studies.

Received: December 15, 2012 Published online: January 24, 2013

[1] R. A. Andersen, E. J. Hwang, G. H. Mulliken, Annu. Rev. Psychol. 2010, 61, 169.

[2] S. Venkatraman, K. Elkabany, J. D. Long, Yimin Yao, J. M. Carmena, IEEE Trans. Bio-Med. Eng. 2009, 56, 15.

[3] G. Fayad, B. Elmiyeh, in Artificial Organs (Ed: N. S. Hakim), Springer, London 2009, pp. 133-136.

[4] X. Liu, D. B. McCreery, R. R. Carter, L. A. Bullara, T. G. Yuen, W. F. Agnew, IEEE Trans. Rehabil. Eng. 1999, 7, 315.

[5] I. Porada, I. Bondar, W. Spatz, J. Krüger, J. Neurosci. Meth. 2000, 95, 13.

[6] R. W. Griffith, D. R. Humphrey, Neurosci. Lett. 2006, 406, 81.

[7] V. S. Polikov, P. A. Tresco, W. M. Reichert, J. Neurosci. Meth. 2005, $148,1$.

[8] D. H. Szarowski, M. D. Andersen, S. Retterer, A. J. Spence, M. Isaacson, H. G. Craighead, J. N. Turner, W. Shain, Brain Res. 2003, 983, 23.

[9] M. T. Fitch, J. Silver, Exp. Neurol. 2008, 209, 294.

[10] J. P. Frampton, M. R. Hynd, J. C. Williams, M. L. Shuler, W. Shain, J. Neural Eng. 2007, 4, 399.

[11] J. Subbaroyan, D. C. Martin, D. R. Kipke, J. Neural Eng. 2005, 2, 103.

[12] H. Lee, R. V. Bellamkonda, W. Sun, M. E. Levenston, J. Neural Eng. $2005,2,81$.
[13] D. K. Cullen, C. M. Simon, M. C. LaPlaca, Brain Res. 2007, 1158, 103.

[14] J. Thelin, H. Jörntell, E. Psouni, M. Garwicz, J. Schouenborg, N. Danielsen, C. E. Linsmeier, PLoS ONE 2011, 6, e16267.

[15] R. Biran, D. C. Martin, P. A. Tresco, J. Biomed. Mater. Res. A 2007, 82, 169.

[16] J. P. Harris, J. R. Capadona, R. H. Miller, B. C. Healy, K. Shanmuganathan, S. J. Rowan, C. Weder, D. J. Tyler, J. Neural Eng. 2011, 8, 066011.

[17] P. J. Rousche, D. S. Pellinen, D. P. Pivin, J. C. Williams, R. J. Vetter, D. R. Kipke, IEEE Trans. Bio-Med. Eng. 2001, 48, 361.

[18] A. Mercanzini, K. Cheung, D. L. Buhl, M. Boers, A. Maillard, P. Colin, J.-C. Bensadoun, A. Bertsch, P. Renaud, Sens. Actuators, A 2008, 143, 90 .

[19] A. E. Hess, J. R. Capadona, K. Shanmuganathan, L. Hsu, S. J. Rowan, C. Weder, D. J. Tyler, C. A. Zorman, J. Micromech. Microeng. 2011, 21, 054009.

[20] Y. Kato, I. Saito, T. Hoshino, T. Suzuki, K. Mabuchi, 28th Annual International Conference of the IEEE Engineering in Medicine and Biology Society 2006, pp. 660-663.

[21] S. Takeuchi, D. Ziegler, Y. Yoshida, K. Mabuchi, T. Suzuki, Lab Chip 2005, 5, 519.

[22] T. Suzuki, K. Mabuchi, S. Takeuchi, in Proc. First International IEEE EMBS Conference on Neural Engineering 2003; pp. 154-156.

[23] B. A. Wester, R. H. Lee, M. C. LaPlaca, J. Neural Eng. 2009, 6, 024002.

[24] J. P. Seymour, D. R. Kipke, Biomaterials 2007, 28, 3594.

[25] S. L. Chorover, A.-M. Deluca, Physiol. Behav. 1972, 9, 671.

[26] G. Lind, C. E. Linsmeier, J. Thelin, J. Schouenborg, J. Neural Eng. 2010, 7, 046005

[27] P. Stice, A. Gilletti, A. Panitch, J. Muthuswamy, J. Neural Eng. 2007, $4,42$.

[28] D. Lewitus, K. L. Smith, W. Shain, J. Kohn, Acta Biomater. 2011, 7, 2483.

[29] J. P. Harris, A. E. Hess, S. J. Rowan, C. Weder, C. A. Zorman, D. J. Tyler, J. R. Capadona, J. Neural Eng. 2011, 8, 046010.

[30] Y. Zhong, G. C. McConnell, J. D. Ross, S. P. DeWeerth, R. V. Bellamkonda, in 2nd International IEEE EMBS Conference on Neural Engineering, 2005, pp. 522-525.

[31] Y. Zhong, R. V. Bellamkonda, Brain Res. 2007, 1148, 15.

[32] D.-H. Kim, D. C. Martin, Biomaterials 2006, 27, 3031.

[33] A. Mercanzini, S. Reddy, D. Velluto, P. Colin, A. Maillard, J.-C. Bensadoun, A. Bertsch, J. A. Hubbell, P. Renaud, In 29th Annual International Conference of the IEEE Engineering in Medicine and Biology Society, 2007, pp. 6612-6615.

[34] L. Spataro, J. Dilgen, S. Retterer, A. J. Spence, M. Isaacson, J. N. Turner, W. Shain, Exp. Neurol. 2005, 194, 289.

[35] Y. Zhong, R. V. Bellamkonda, J. Controlled Release 2005, 106, 309.

[36] E. K. Purcell, D. E. Thompson, K. A. Ludwig, D. R. Kipke, J. Neurosci. Meth. 2009, 183, 149.

[37] E. Azemi, C. F. Lagenaur, X. T. Cui, Biomaterials 2011, 32, 681.

[38] S. David, S. Lacroix, Annu. Rev. Neurosci. 2003, 26, 411.

[39] B. K. Kwon, E. B. Okon, W. Plunet, D. Baptiste, K. Fouad, J. Hillyer, L. C. Weaver, M. G. Fehlings, W. Tetzlaff, J. Neurotraum. 2011, 28, 1589.

[40] N. J. Tester, A. H. Plaas, D. R. Howland, J. Neurosci. Res. 2007, 85, 1110.

[41] H. Lee, R. J. McKeon, R. V. Bellamkonda, Proc. Natl. Acad. Sci. USA 2010, 107, 3340 .

[42] M. Nazari-Robati, K. Khajeh, M. Aminian, M. Fathi-Roudsari, A. Golestani, Int. J. Biol. Macromol. 2012, 50, 487.

[43] G. H. Altman, F. Diaz, C. Jakuba, T. Calabro, R. L. Horan, J. Chen, H. Lu, J. Richmond, D. L. Kaplan, Biomaterials 2003, 24, 401.

[44] L. Meinel, S. Hofmann, V. Karageorgiou, C. Kirker-Head, J. McCool, G. Gronowicz, L. Zichner, R. Langer, G. Vunjak-Novakovic, D. L. Kaplan, Biomaterials 2005, 26, 147. 
[45] X. Hu, K. Shmelev, L. Sun, E.-S. Gil, S.-H. Park, P. Cebe, D. L. Kaplan, Biomacromolecules 2011, 12, 1686.

[46] Y. Wang, D. D. Rudym, A. Walsh, L. Abrahamsen, H.-J. Kim H. S. Kim, C. Kirker-Head, D. L. Kaplan, Biomaterials 2008, 29, 3415.

[47] D. N. Rockwood, R. C. Preda, T. Yucel, X. Wang, M. L. Lovett, D. L. Kaplan, Nat. Protoc. 2011, 6, 1612.

[48] E. M. Pritchard, P. B. Dennis, F. Omenetto, R. R. Naik, D. L. Kaplan, Biopolymers 2012, 97, 479.

[49] C. Szybala, E. M. Pritchard, T. A. Lusardi, T. Li, A. Wilz, D. L. Kaplan, D. Boison, Exp. Neurol. 2009, 219, 126.

[50] E. M. Pritchard, D. L. Kaplan, Expert Opin. Drug Delivery 2011, 8, 797.

[51] Y.-C. Lin, M. Ramadan, M. Hronik-Tupaj, D. L. Kaplan, B. J. Philips, W. Sivak, J. P. Rubin, K. G. Marra, Ann. Plast. Surg. 2011, 67, 147.

[52] W. Huang, R. Begum, T. Barber, V. Ibba, N. C. H. Tee, M. Hussain M. Arastoo, Q. Yang, L. G. Robson, S. Lesage, T. Gheysens, N. J. V. Skaer, D. P. Knight, J. V. Priestley, Biomaterials 2012, 33, 59.

[53] F. Zhang, R. Liu, B. Q. Zuo, J. Z. Qin, in 4th International Conference on Bioinformatics and Biomedical Engineering (iCBBE), IEEE, 2010, pp. 1-4.

[54] Y. Yang, X. Yuan, F. Ding, D. Yao, Y. Gu, J. Liu, X. Gu, Tissue Eng Pt A $2011,17,2231$

[55] S. Madduri, M. Papaloïzos, B. Gander, Biomaterials 2010, 31, 2323.

[56] A. M. Ghaznavi, L. E. Kokai, M. L. Lovett, D. L. Kaplan, K. G. Marra, Ann. Plast. Surg. 2011, 66, 273.

[57] V. Benfenati, S. Toffanin, R. Capelli, L. M. A. Camassa, S. Ferroni, D. L. Kaplan, F. G. Omenetto, M. Muccini, R. Zamboni, Biomaterials 2010, 31, 7883

[58] C. R. Wittmer, T. Claudepierre, M. Reber, P. Wiedemann, J. A. Garlick, D. Kaplan, C. Egles, Adv. Funct. Mater. 2011, 21, 4232.

[59] D.-H. Kim, J. Viventi, J. J. Amsden, J. Xiao, L. Vigeland, Y.-S. Kim, J. A. Blanco, B. Panilaitis, E. S. Frechette, D. Contreras, D. L. Kaplan, F. G. Omenetto, Y. Huang, K.-C. Hwang, M. R. Zakin, B. Litt, J. A. Rogers, Nat. Mater. 2010, 9, 511.

[60] A. A. Sharp, A. M. Ortega, D. Restrepo, D. Curran-Everett, K. Gall, IEEE Trans. Bio-Med. Eng. 2009, 56, 45.
[61] V. S. Polikov, M. L. Block, J.-M. Fellous, J.-S. Hong, W. M. Reichert, Biomaterials 2006, 27, 5368.

[62] V. S. Polikov, E. C. Su, M. A. Ball, J.-S. Hong, W. M. Reichert, J. Neurosci. Meth. 2009, 181, 170.

[63] A. K. H. Achyuta, V. S. Polikov, A. J. White, H. G. P. Lewis, S. K. Murthy, Macromol. Biosci. 2010, 10, 872.

[64] J. L. Skousen, S. M. E. Merriam, O. Srivannavit, G. Perlin, K. D. Wise, P. A. Tresco, Prog. Brain Res. 2011, 194, 167.

[65] C. Jiang, X. Wang, R. Gunawidjaja, Y.-H. Lin, M. K. Gupta, D. L. Kaplan, R. R. Naik, V. V. Tsukruk, Adv. Funct. Mater. 2007, 17, 2229.

[66] X. Wang, H. J. Kim, P. Xu, A. Matsumoto, D. L. Kaplan, Langmuir 2005, 21, 11335 .

[67] Q. Lu, X. Hu, X. Wang, J. A. Kluge, S. Lu, P. Cebe, D. L. Kaplan, Acto Biomater. 2010, 6, 1380.

[68] B. D. Lawrence, S. Wharram, J. A. Kluge, G. G. Leisk, F. G. Omenetto, M. I. Rosenblatt, D. L. Kaplan, Macromol. Biosci. 2010, 10, 393.

[69] K. Najafi, J. F. Hetke, IEEE Trans. Bio-Med. Eng. 1990, 37, 474.

[70] C. S. Bjornsson, S. J. Oh, Y. A. Al-Kofahi, Y. J. Lim, K. L. Smith, J. N. Turner, S. De, B. Roysam, W. Shain, S. J. Kim, J. Neural. Eng. 2006, 3, 196.

[71] Y.-C. Huang, S.-H. Hsu, M.-T. Chen, C.-H. Hsieh, W.-C. Kuo, H. Cheng, Y.-Y. Huang, Carbohyd. Polym. 2011, 84, 788

[72] T. Liu, J. Xu, B. P. Chan, S. Y. Chew, J. Biomed. Mater. Res., A 2012, 100A, 236.

[73] Y. Xia, G. M. Whitesides, Angew. Chem. Int. Ed. 1998, 37, 550.

[74] F. Wu, M. Im, E. Yoon, in 16th International Solid-State Sensors, Actuators and Microsystems Conference (TRANSDUCERS), 2011, pp. 966-969.

[75] R. Hoffmann, T. Stieglitz, N. H. Hosseini, S. Kisban, O. Paul, P. Ruther, in 29th Annual International Conference of the IEEE Engineering in Medicine and Biology Society, 2007, pp. 4711-4714.

[76] B. D. Lawrence, F. Omenetto, K. Chui, D. L. Kaplan, J. Mater. Sci. 2008, 43, 6967

[77] L. W. Tien, E. S. Gil, S.-H. Park, B. B. Mandal, D. L. Kaplan, Macromol. Biosci. 2012, 12, 1671 .

[78] R. W. Farndale, D. J. Buttle, A. J. Barrett, BBA-Gen. Subjects 1986 883,173 